\title{
Vitreous humour and cerebrospinal fluid hypoxanthine concentration as a marker of pre-mortem hypoxia in SIDS
}

\author{
K H Carpenter, J R Bonham, E Worthy, S Variend
}

\begin{abstract}
Aims-To assess the rate at which premortem hypoxia occurs in sudden infant death syndrome (SIDS) when compared with death in early childhood.

Methods-The hypoxanthine concentration was measured as a marker of premortem hypoxia in vitreous humour and cerebrospinal fluid samples obtained at necropsy from 119 children whose ages ranged from 1 week to 2 years.
\end{abstract}

Results-Increasing interval between death and necropsy was accompanied by an increase in the hypoxanthine concentration of vitreous humour for the first 24 hours, at a rate of $8 \cdot 3 \mu \mathrm{mol} / \mathrm{l} / \mathrm{hour}$. Thereafter, there was little change with time, and the results were corrected to 24 hours according to a regression equation. Cerebrospinal fluid concentrations showed no significant change with time following death. Patients were divided into three groups according to the cause of death: SIDS, cardiac or pulmonary disease, and others. Median values for the cerebrospinal fluid hypoxanthine concentrations were not significantly different among the groups and no difference could be shown between the vitreous humour hypoxanthine concentration in cases of SIDS and those children dying from other causes. Patients with established cardiac or pulmonary disease had a significantly reduced vitreous humour hypoxanthine concentration which may have reflected the premortem use of artificial ventilation.

Conclusions-The results of this study do not support the view that pre-mortem hypoxia is a common feature in SIDS when compared with other causes of death.

(F Clin Pathol 1993;46:650-653)

Department of Chemical Pathology, Children's Hospital,

Western Bank, Sheffield S10 2TH K H Carpenter J R Bonham

E Worthy

$S$ Variend

Correspondence to:

Dr J R Bonham

Accepted for publication 20 January 1993 attention has focused on the use of hypoxanthine as a marker of pre-mortem hypoxia ${ }^{1}$ and initial reports have suggested a higher concentration of hypoxanthine in the vitreous humour cases of SIDS when compared with those of controls. ${ }^{2}$ Unfortunately, studies using animals have cast doubt on the validity of these findings due to the rapid increase in hypoxanthine concentration in vitreous humour following death. ${ }^{34}$

Hypoxanthine concentrations in cerebrospinal fluid have also been shown to increase during severe hypoxia, ${ }^{5}$ but concentrations after death have not been studied.

\section{Methods}

Vitreous humour was sampled at 119 necropsies in children whose ages ranged from 1 week to 2 years. The cause of death was unexplained in 68 cases (SIDS) and explained in 51. Of these 51, 13 died with severe cardiac or pulmonary disease, thought likely to be associated with pre-mortem hypoxia. The remainder died from a variety of conditions. CSF was obtained in 75 cases, 45 of which were cases of SIDS. Samples were obtained $2 \cdot 5-120$ hours after death and were stored at $-20^{\circ} \mathrm{C}$ before analysis.

The hypoxanthine analysis was performed by a high performance liquid chromatography (HPLC) technique using a modification of the method described by Morris et al. ${ }^{6}$ Samples were diluted 1 in 1 with $10 \%$ weight/volume trichloroacetic acid containing $100 \mathrm{mg} / 1$ allopurinol as an internal standard. The protein precipitate was removed by centrifugation and the supernatant fluid was washed three times with equal volumes of water saturated diethyl ether. Washed supernatant fluid $(20 \mu \mathrm{l})$ was applied to a $25 \mathrm{~cm} \times$ $4.6 \mathrm{~mm}$ ODS 2 reverse phase column (Phase Separations Limited, Deeside, England). Gradient elution with a flow rate of 1 $\mathrm{ml} /$ minute was used; the equipment comprised a Model 425 gradient former and Model 420 pump (Kontron Instruments Limited, Watford England). The mobile phase ranged from $100 \%$ solvent A $(40 \mathrm{mM}$ ammonium acetate containing $1 \% \quad \mathrm{v} / \mathrm{v}$ methanol, adjusted to $\mathrm{pH} 5 \cdot 0$, with glacial acetic acid) by a linear gradient to $15 \%$ solvent B ( $80 \%$ methanol, $10 \%$ acetonitrile, $10 \%$ tetrahydrofuran $\mathrm{v} / \mathrm{v}$ ) over 20 minutes. Column eluent was monitored at 254 and $280 \mathrm{~nm}$ on a Gilson Model 116 detector (Anachem, Luton, England).

Correlation analysis was performed using the Spearman rank sum test, with the regression equation calculated by least squares analysis. Comparison between groups was done by the Mann-Witney U test. 
Figure 1 Hypoxanthine concentration measured in vitreous humour obtained from 119 necropsies performed in children aged between one week and two years. There is a significant correlation between hypoxanthine concentration and post mortem interval up to 24 hours; beyond this time, no significant correlation can be shown.
Figure 2 Hypoxanthine concentration measured in cerebrospinal fluid obtained from 75 necropsies performed in children aged between 1 week and 2 years. The results show no significant correlation with post mortem interval.

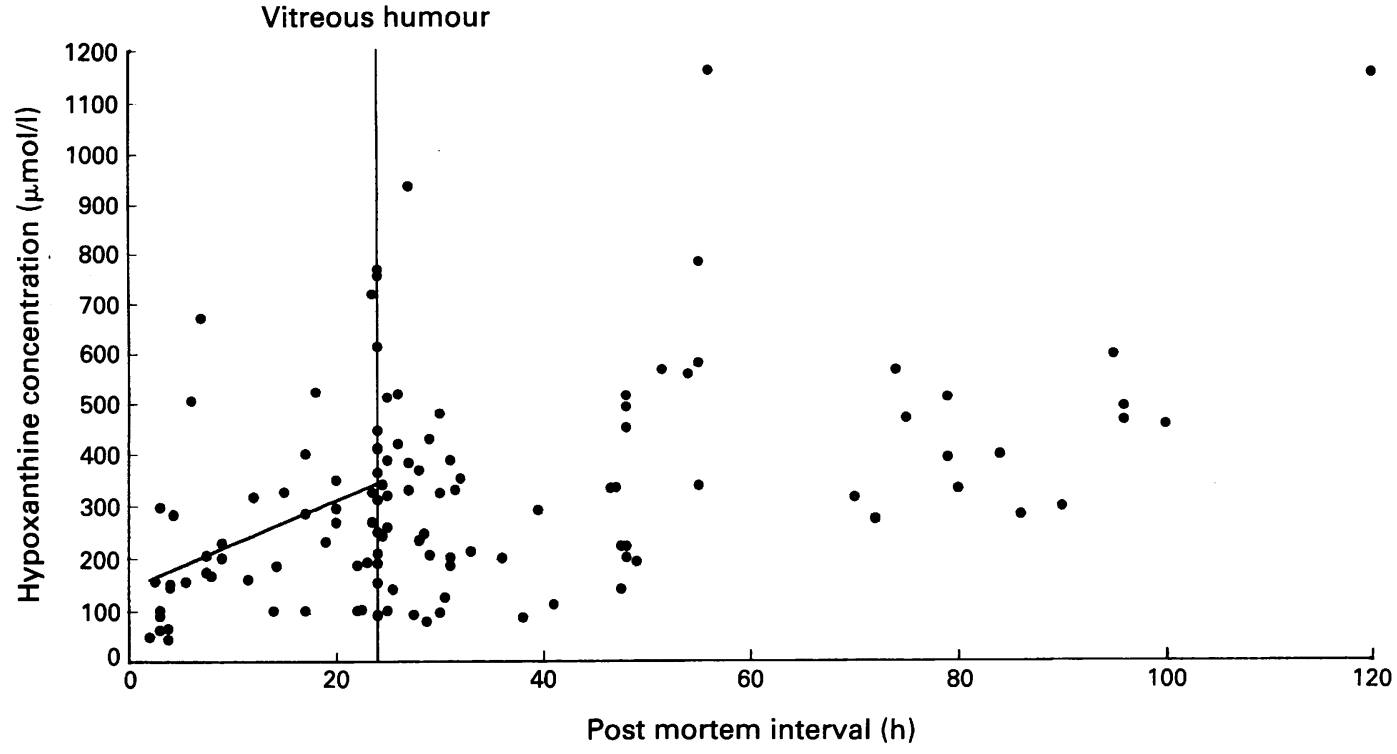

\section{Results}

During the first 24 hours after death vitreous humour hypoxanthine concentrations showed a significantly positive correlation with time. The regression equation showed that the hypoxanthine concentration increased at a mean rate of $8.3 \mu \mathrm{mol} / /$ hour, correlation coefficient $\mathrm{r}=0.46 ; \mathrm{p}<0.001$ (fig 1). Beyond 24 hours, the concentration of hypoxanthine in the vitreous humour was weakly correlated with post mortem interval; correlation coefficient $\mathrm{r}=0.32 ; \mathrm{p}=0.010$. In all subsequent comparisons results obtained from cases with a post mortem interval of less than 24 hours were corrected to a 24 hour interval equivalent according to the regression equation.

Correlation analysis with the Spearman rank sum test applied to hypoxanthine concentrations in the cerebrospinal fluid showed no significant correlation with post mortem interval over any time period (fig 2 ).
In the three groups studied the median concentration of hypoxanthine in vitreous humour, when normalised to a 24 hour equivalent post mortem interval was: 338 $\mu \mathrm{mol} / 1$ in SIDS cases; $264 \mu \mathrm{mol} / 1$ in the cardiac/pulmonary disease group; and 295 $\mu \mathrm{mol} / 1$ in those cases resulting from other causes of death. In the corresponding groups the median cerebrospinal fluid hypoxanthine concentration was: $884 \mu \mathrm{mol} / \mathrm{l} ; 796 \mu \mathrm{mol} / \mathrm{l}$; and $796 \mu \mathrm{mol} / 1$, respectively. The distribution of these results obtained is shown in figs 3 and 4.

Analysis using the Mann-Witney $U$ test showed no significant differences among the three groups when applied to cerebrospinal fluid. The vitreous humour hypoxanthine results showed no significant difference when the SIDS cases and those resulting from other causes of death were compared. The vitreous humour hypoxanthine concentration in children who died with established cardiac or

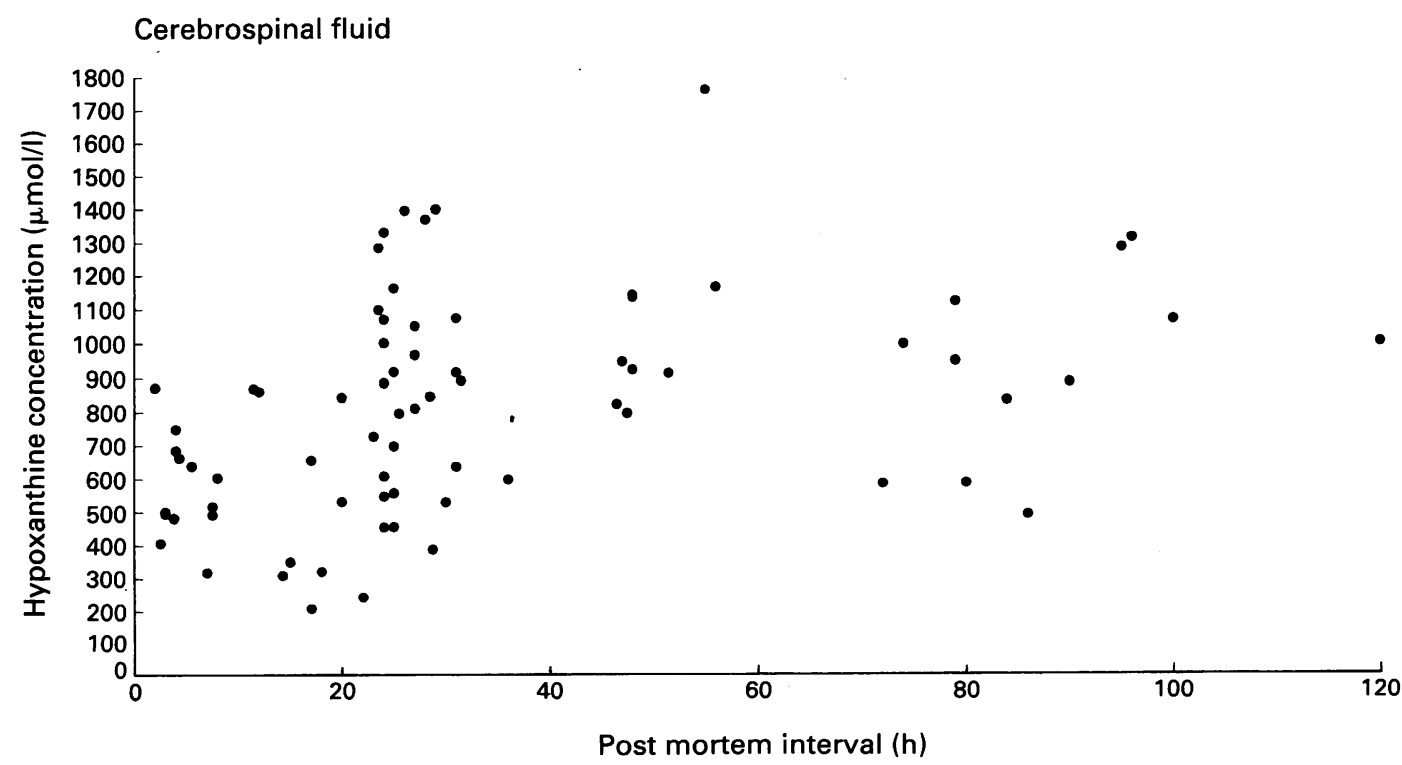


Figure 3 Distribution of hypoxanthine concentration in vitreous humour obtained at necropsy from children aged between 1 week and 2 years dying with: cardiac or pulmonary disease, SIDS and other causes.
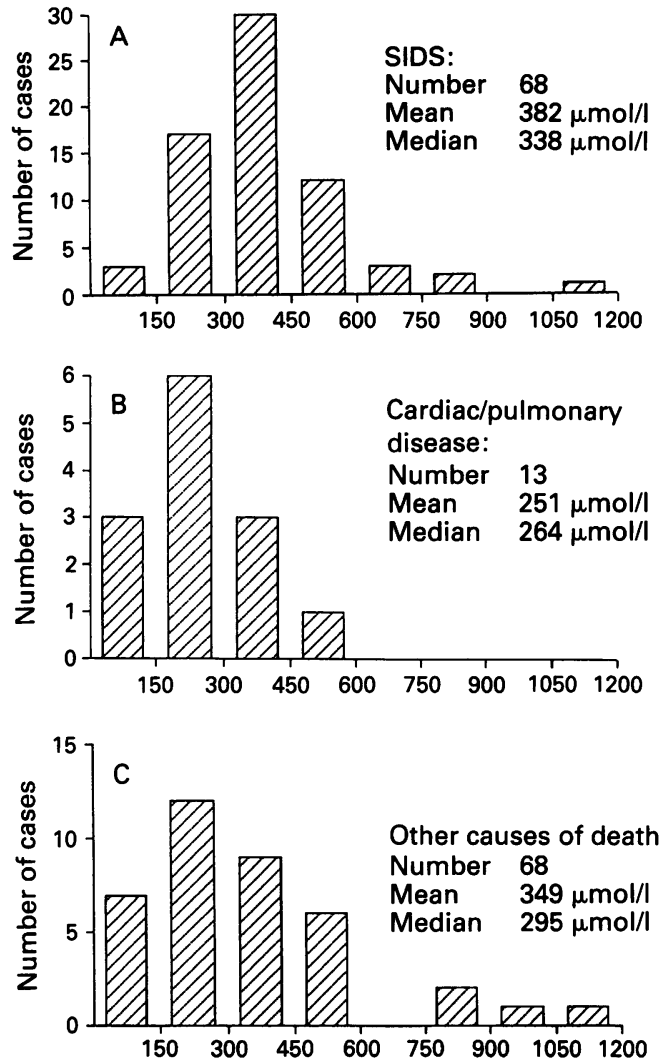

Hypoxanthine concentration ( $\mu \mathrm{mol} / \mathrm{l})$

Figure 4 Distribution of hypoxanthine concentration in cerebrospinal fluid obtained at necropsy from children aged between 1 week and 2 years dying with: cardiac or pulmonary disease, SIDS, and other causes.
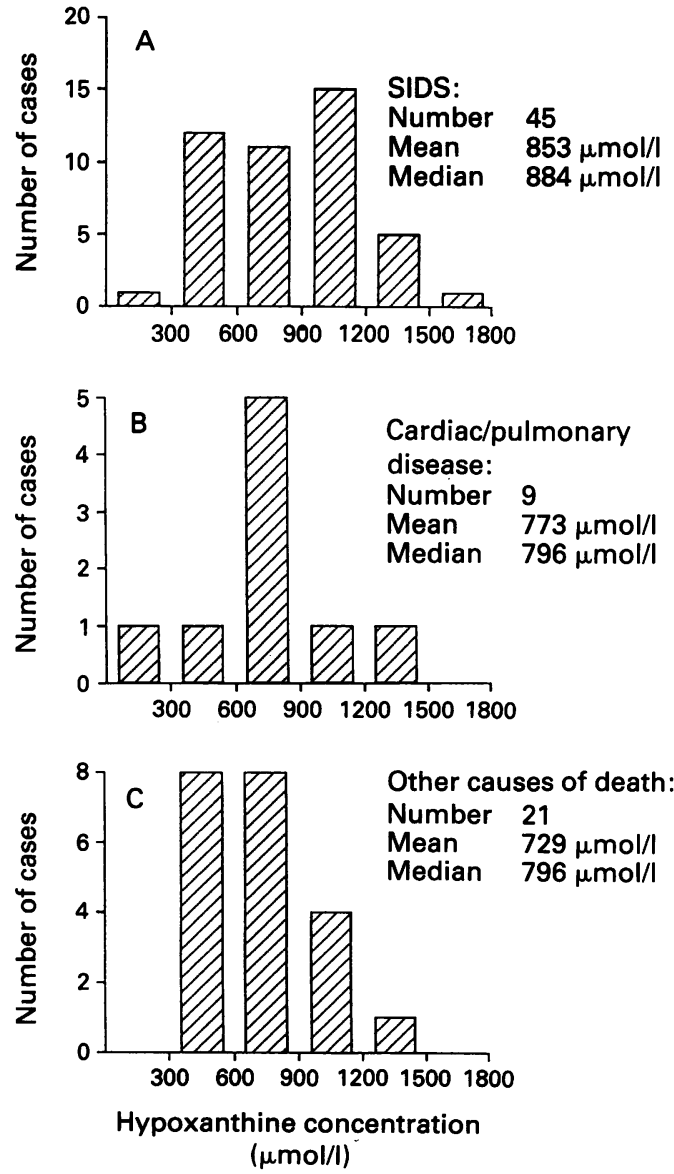

pulmonary disease was reduced however, when compared with SIDS cases; $\mathrm{p}=0.007$.

\section{Discussion}

Several previous reports have shown a post mortem increase in the hypoxanthine concentration of vitreous humour in experimental animals. In 6 week old chickens Gardiner et al found that the vitreous humour hypoxanthine concentration increased from 16 to $263 \mu \mathrm{mol} / 1(10.3 \mu \mathrm{mol} / 1 /$ hour $)$ when the dead chicks were stored at $20^{\circ} \mathrm{C}$, and from zero to $109 \mu \mathrm{mol} / 1 \quad(4.5 \mu \mathrm{mol} / 1 /$ hour $)$ when maintained at $4^{\circ} \mathrm{C}$ over 24 hours. ${ }^{3}$ These increases were continued beyond 24 hours and the rate of hypoxanthine accumulation increased up to 120 hours post mortem.

In the same paper the authors reported a post mortem increase in vitreous humour hypoxanthine concentration in pigs from a mean of $15 \mu \mathrm{mol} / 1$ at zero hour to $329 \mu \mathrm{mol} / 1$ at 24 hours at a rate of $13 \mu \mathrm{mol} / 1 /$ hour; the storage temperature was $20^{\circ} \mathrm{C}$. Poulson et al demonstrated changes in vitreous humour from pigs averaging $4.3 \mu \mathrm{mol} / 1 /$ hour but with a wide range $(1 \cdot 25-8 \mu \mathrm{mol} / \mathrm{l} / \mathrm{hour}$, even when the storage temperature was controlled at $6^{\circ} \mathrm{C}$. ${ }^{4}$ The same authors reported a mean post mortem increase in hypoxanthine concentration of human vitreous humour of 3.5-4 $\mu \mathrm{mol} / 1 /$ hour.

The findings of this study support the observation that hypoxanthine accumulates in the vitreous humour after death. The rate of increase observed in the first 24 hours, $8.3 \mu \mathrm{mol} / 1 /$ hour, is close to the average reported in previous studies. After 24 hours, only a weak correlation between hypoxanthine concentration and post mortem interval could be shown. No correlation between hypoxanthine concentration in the cerebrospinal fluid and post mortem interval was observed.

Initial reports of hypoxanthine measurement in vitreous humour obtained post mortem compared cases of sudden death due to trauma, strangulation, and myocardial infarction with cases of fatal drug overdose. ${ }^{1}$ These results indicated an increased hypoxanthine concentration in people dying from an overdose of respiratory depressant drugs likely to cause pre-mortem hypoxia.

When investigating the possible role of premortem hypoxia in SIDS, Rognum et al compared the hypoxanthine concentration in vitreous humour from SIDS cases with a small group dying from other causes. ${ }^{2}$ The median concentration in SIDS cases $(380 \mu \mathrm{mol} / \mathrm{l})$ was significantly higher $(\mathrm{p}<$ $0.001)$ than the median $(53 \mu \mathrm{mol} / \mathrm{l})$ in the group dying from other causes. While the results which we obtained in the SIDS cases (median $338 \mu \mathrm{mol} / \mathrm{l}$ ) compared very closely with those of Rognum et al our findings do not substantiate a significant difference from non-SIDS cases in which the median was $295 \mu \mathrm{mol} / 1$.

Cases with known cardiac or pulmonary disease, in whom hypoxia may have been 
expected, were separated from the control group. In practice, however, the use of artificial ventilation in these patients probably prevented the development of tissue hypoxia; indeed, the vitreous humour hypoxanthine concentration in this group was less than in the remaining two groups (figs 3 and 4). The close comparison between the results obtained in the SIDS group and the remaining non-SIDS cases does not support the view that in most cases SIDS is associated with a prolonged period of respiratory insufficiency, as proposed by Rognum et al. ${ }^{2}$

The overall concentration of hypoxanthine in cerebrospinal fluid in this study was higher than that found in vitreous humour. This may reflect the high energy consumption of the infant brain, resulting in a rapid release of hypoxanthine at the time of death, or be due to the low xanthine oxidase activity of the human brain, ${ }^{7}$ leading to a slow clearance of accumulated hypoxanthine. Again, no statisti- cal difference could be demonstrated between SIDS and non-SIDS cases.

1 Saugstad OD, Olaisen B. Post-mortem hypoxanthine levels in the vitreous humour. An introductory report. Forensic Sci Int 1978;12:33-6.

2 Rognum TO, Saugstad OD, Oyasaeter S, Olaisen B. Elevated levels of hypoxanthine in vitreous humour indicate prolonged cerebral hypoxia in victims of sudden infant death. Pediatrics 1988;82:615-7.

3 Gardiner EE, Newberry RC, Keng JY. Postmortem time and storage temperature affect the concentrations of hypoxanthine, other purines, pyrimidines, and nucleosides in avian and porcine vitreous humour. Pediatr Res 1989;26:639-42.

4 Poulsen JP, Rognum TO, Oyasaeter S, Saugstad OD Changes in oxypurine concentrations in vitreous humour of pigs during hypoxaemia and post-mortem. humour of pigs during hypox
Pediatr Res 1990;28:482-4.

5 Lun A, Gross J, Siggel H, Pohle R. Concentration of hypoxanthine in both cerebrospinal fluid and brain tissue increases under intensive hypoxia only. Biol Neonate 1988;54:195-202.

6 Morris GS, Simmonds HA, Davies PM. Use of biological fluids for the rapid diagnosis of potentially lethal disorders of human purine and pyrimidine metabolism. Biomed Chromatogr 1986;3:109-18.

7 Parks DA, Granger DN. Xanthine oxidase: biochemistry, distribution and physiology. Acta Physiol Scand (Suppl) 1986;548:87-99. 